Research Article

\title{
Coheritability and Genetic Advances of Agromorphological and Oil Quality Traits in Groundnut (Arachis hypogaea L.) Genotypes from Ethiopia
}

\author{
Zekeria Yusuf $\mathbb{D}^{1}{ }^{1}$ Wassu Mohammed, ${ }^{2}$ Habtamu Zeleke, ${ }^{2}$ Shimelis Hussein, ${ }^{3}$ \\ and Hugo Arno ${ }^{4}$ \\ ${ }^{1}$ Biology Department, Haramaya University, Dire Dawa, Ethiopia \\ ${ }^{2}$ School of Plant Science, Haramaya University, Dire Dawa, Ethiopia \\ ${ }^{3}$ Department of Crop Science, University of Kwazulu-Natal, Durban, South Africa \\ ${ }^{4}$ Department of Food Science, University of Free State, Bloemfontein, South Africa
}

Correspondence should be addressed to Zekeria Yusuf; zakoyusuf@yahoo.com

Received 16 September 2021; Revised 13 October 2021; Accepted 13 December 2021; Published 24 December 2021

Academic Editor: Mehdi Rahimi

Copyright $\odot 2021$ Zekeria Yusuf et al. This is an open access article distributed under the Creative Commons Attribution License, which permits unrestricted use, distribution, and reproduction in any medium, provided the original work is properly cited.

Groundnut (Arachis hypogaea L.) is one of the most important oils, food, confectionery, and feed crops due to its high oil quality and richness in all classes of nutrients. Coheritability helps in determining the pattern of coinheritance of two or more traits. This study was aimed at determining the coheritability of oil and yield traits to provide means of conducting improvement of oil traits through breeding for yield traits. The coheritabilities of the traits were estimated by a pooled analysis of covariance (ANCOVA) for agromorphological and oil traits. The result indicated that all oil traits have shown medium to high coheritability with oil content, oil quality, and seed yield. The coheritability and genetic advance of the three agronomically important traits in oil crops including oil content, oleic to linoleic acid (O/L) ratio, and seed yield (SY) is greater than oil content, linoleic acid, and SY can be further evidence showing that both oil content and oil quality traits can be improved with seed yield, indicating that there is the possibility of making a selection for genotypes with high oil content, oil yield, and oil quality with high seed yield traits.

\section{Introduction}

Groundnut or peanut (Arachis hypogaea L.) is an important oil, food, and feed legume crop [1]. The kernels are rich in oil $(48-50 \%)$ and protein $(25-28 \%)$ and are the source of several vitamins, minerals, and biologically active compounds such as polyphenols, flavonoids, and isoflavones. Thus, groundnut and products based on groundnut can be promoted as nutritional foods to fight energy, protein, and micronutrient malnutrition among the poor [2]. Groundnut is mainly cultivated as a source of vegetable oil for local, regional, and international markets [3]. Groundnut genotypes with oleic acid content $\geq 78 \%$ are referred to as high oleic genotypes and possess oil with longer shelf-life $[4,5]$. High oleic acid/linoleic acid ratio is the desired quality parameter to enhance the shelf-life of oil seeds. Developing groundnut genotypes with high oleic acid is a key breeding objective for human health and product quality and to access the lucrative market opportunities [6]. The groundnut shells are used for making particle boards or used as fuel or filler in the fertilizer and feed industry. Groundnut haulms constitute nutritious fodder for livestock. Groundnut seed can be consumed raw (nonheated), boiled, and roasted and also used to make confections and its flour to make baked products [2]. Groundnut production in Ethiopia is found to be constrained by several biotic and abiotic factors like critical moisture stress, especially during flowering and after, lack of improved varieties, inappropriate production and postharvest practices, diseases, and pests [7].

The phenotypes of a trait are often the result of a complex interaction of multiple genetic and environmental factors [8], and the ability to assay such traits presents a unique 
opportunity to explore mechanisms underlying concerted inheritance. Heritability is a measure of the expression of a genetic trait. The estimates of heritability alone do not indicate the associating genetic progress that may result from choosing better performing plants. The heritability together with phenotypic variances and the selection intensity, however, promise the estimation of genetic advance or response to selection that is more useful in the selection of promising genotypes [9-11]. The term coheritability was originally coined by Nei [12] as a measure of joint inheritance of two or more traits that represent the genetic contribution to their phenotypic correlation. Falconer and MacKay [13] denoted coheritability as a correlated response to selection.

Despite the various terms and methods used for measuring it [14], the coheritability estimate is being one of the basic parameters for measuring the coinheritance of traits [15]. The advantage of this genetic parameter over the genetic correlation coefficient is quite obvious as the latter throws light only on the magnitude of relationship among traits in the existing generation and not their coinheritance $[16,17]$. The joint analysis of traits provides the greater statistical power to detect the causal genetic factors for the association of traits [18-20]. Breeding for oil traits is difficult since they are usually associated with low heritability. One means of conducting breeding for such traits is making indirect selection through highly heritable seed yield traits. Furthermore, few studies were conducted on the coheritability of oil traits. Therefore, this study is undertaken to estimate coheritability estimates for agromorphological and oil traits. This can offer a useful functional understanding of relevant systems to underpin future groundnut breeding programs and products development.

\section{Materials and Methods}

The experimental materials consisted of fifteen groundnut genotypes obtained from Werer Agricultural Research Center, Ethiopia, and a local check variety. The field experiment was carried out across four locations, namely, Fedis, Mechara, Pawe, and Guba in Ethiopia under rain-fed conditions. The experimental design was a completely randomized block design in three replications. The groundnut seeds were planted and the treatments were given the required agronomic practices including weeding, hoeing, fertilizer application, and the necessary plant protection. Before running the laboratory experiment, the moisture content of seed samples was reduced to $5 \%$. The lab experiment was carried out in two replications by taking $10 \mathrm{gm}$ of seed samples from 16 groundnut genotypes. Oil content and fatty acid profile determination were carried out according to Folch et al. [21]. Iodine value was determined with the Hanus methodology [22].

Field experimental data were recorded for twelve agromorphological traits, namely, number of mature pods per plant (NMP), number of primary branches per plant
(NBP), above-ground biomass per plant (AGBP, g), pod weight per plant (PWP, g), number of seeds per plant (NSP), seed weight per plant (SWP, g), hundred seed weight (100SW, g), number of seeds per pod (NSPOD), and seed yield per hectare (SY, $\mathrm{kg} / \mathrm{ha})$. Data for fatty acid composition were collected for seventeen oil traits including oil content, iodine value (IV), palmitic acid, stearic acid, oleic acid, linoleic acid, arachidic acid, eicosenoic acid, behenic acid, and lignoceric acid; S: total saturated fatty acids; MUS: total monounsaturated fatty acids; PUS: total polyunsaturated fatty acids; US: total unsaturated fatty acids; PUS/S: total polyunsaturated to saturated fatty acids; O/L: oleic to linoleic acid ratio; and OY: oil yield $(\mathrm{kg} / \mathrm{ha})$.

The coheritability of the traits was estimated by analysis of covariance (ANCOVA) based on the mean sum of products.

2.1. Estimation of Genotypic and Phenotypic Covariance. Genotypic and phenotypic covariance was estimated by using the following formulae:

$$
\text { genotypic covariance }\left(\sigma g_{x} g_{y}\right)=\frac{\mathrm{MSP}_{v}-\mathrm{MSP}_{e}}{r L},
$$

where $\mathrm{MSP}_{V}=$ mean sum of products of traits $x, y$, and/or $z \ldots$...; $\mathrm{MSP}_{e}=$ mean sum of products due to error and $r=$ number of replications; and $L$ is the number of locations.

$$
\text { Phenotypic covariance }\left(\sigma p_{x} p_{y}\right)=\sigma p_{x} p_{y}+\sigma e_{x} e_{y},
$$

where $\sigma g_{x} g_{y}=$ genotypic covariance; and $\sigma e_{x} e_{y}=$ mean sum of products due to error of traits $x$ and $y$.

2.2. Estimation of Coheritability. Coheritability values were estimated according to the following formulae:

$$
\text { coheritability }=\frac{\text { genotypic covariance }}{\text { phenotypic covariance }} \text {. }
$$

The heritability percentage was categorized as low when less than $40 \%$, medium, $40-59 \%$, moderately high, $60-79 \%$, and very high, $80 \%$ and above, as indicated by Comstock and Robinson [23].

Genetic advance (GA) was calculated with the method suggested by Allard [24] and Singh and Chaudhury [25]: $\mathrm{GA}=K \sigma_{p} H^{2}$, where GA: genetic advance; $K$ : constant $=1.76$ at $10 \%$ selection intensity; $\sigma_{p}$ : standard deviation of phenotypic variance; and $H^{2}$ : heritability in broad sense. $\mathrm{GA}$ as $\%$ of mean $(\mathrm{GAM})=(\mathrm{GA} / \bar{x}) \times 100 \%$. The genetic advance (GA), expressed as a percentage of mean, was categorized as high when it is above $20 \%$, moderate, $10-20 \%$, and low when it is less than $10 \%$ based on Johnson et al. [26]. The data in the supplementary file were subjected to combined analysis of covariance (ANOVA) using SAS Proc Mixed procedure [27] with genotypes being considered as fixed effects, whereas locations, replications, and blocks within locations as random effects. Homogeneity of variance was checked using the Obrien test [28]. 
TABLe 1: Coheritability (above diagonal) with genetic advance (below diagonal) for oil traits and seed yield.

\begin{tabular}{lcccccccccccccccccc}
\hline Trait & Oil & IV & Pal & St & Oleic & Lin & Ara & Eic & Beh & Lig & S & MUS & PUS & US & PUS/S & O/L & SY & OY \\
\hline Oil & & 61 & 94 & 86 & 83 & 86 & 87 & 94 & 92 & 70 & 90 & 85 & 86 & 50 & 65 & 78 & 73 & 74 \\
IV & 4.0 & & 96 & 90 & 94 & 86 & 91 & 90 & 86 & 73 & 96 & 95 & 86 & 77 & 71 & 83 & 65 & 68 \\
Pal & 16 & 19 & & 92 & 87 & 95 & 93 & 90 & 90 & 63 & 98 & 88 & 95 & 97 & 89 & 73 & 68 & 71 \\
Stearic & 39 & 38 & 46 & & 79 & 96 & 82 & 84 & 89 & 64 & 87 & 79 & 96 & 86 & 96 & 65 & 79 & 78 \\
Oleic & 11 & 9.0 & 8.0 & 33 & & 48 & 84 & 97 & 96 & 78 & 81 & 89 & 48 & 92 & 81 & 78 & 79 & 79 \\
Lin & 15 & 18 & 31 & 49 & 3.0 & & 95 & 81 & 77 & 67 & 95 & 48 & 89 & 83 & 76 & 90 & 49 & 55 \\
Ara & 28 & 25 & 31 & 61 & 27 & 31 & & 98 & 92 & 69 & 89 & 84 & 95 & 88 & 94 & 75 & 81 & 81 \\
Eic & 34 & 34 & 28 & 2.0 & 42 & 62 & 53 & & 88 & 88 & 91 & 97 & 81 & 93 & 83 & 97 & 73 & 74 \\
Beh & 20 & 16 & 20 & 51 & 25 & 16 & 44 & 38 & & 80 & 92 & 95 & 77 & 91 & 60 & 91 & 80 & 81 \\
Lig & 20 & 21 & 13 & 23 & 27 & 18 & 21 & 54 & 29 & & 51 & 79 & 67 & 75 & 76 & 71 & 73 & 74 \\
S & 13 & 13 & 27 & 48 & 9.0 & 24 & 37 & 26 & 26 & 12 & & 81 & 95 & 94 & 87 & 74 & 75 & 76 \\
MUS & 12 & 10 & 8.0 & 32 & 22 & 3.0 & 27 & 42 & 25 & 28 & 9.0 & & 48 & 93 & 82 & 79 & 78 & 79 \\
PUS & 15 & 18 & 31 & 49 & 3.0 & 29 & 31 & 29 & 16 & 19 & 24 & 3.0 & & 83 & 76 & 90 & 49 & 55 \\
US & 2.0 & 4.0 & 15 & 35 & 13 & 13 & 25 & 37 & 19 & 23 & 9.0 & 14 & 13 & & 70 & 81 & 70 & 72 \\
PUS/S & 9.0 & 13 & 23 & 38 & 11 & 21 & 21 & 37 & 10 & 26 & 15 & 12 & 21 & 11 & & 94 & 41 & 46 \\
O/L & 24 & 22 & 15 & 34 & 33 & 12 & 36 & 49 & 37 & 34 & 21 & 33 & 12 & 25 & 21 & & 81 & 80 \\
SY & 30 & 24 & 27 & 62 & 37 & 17 & 55 & 37 & 43 & 38 & 35 & 37 & 17 & 28 & 14 & 51 & 64 \\
OY & 32 & 26 & 28 & 62 & 38 & 19 & 56 & 37 & 44 & 38 & 36 & 38 & 19 & 30 & 16 & 51 & 53 \\
\hline
\end{tabular}

IV: iodine value; Pal: palmitic acid; St: stearic acid; Lin: linoleic acid; Ara: arachidic acid; Eic: eicosenoic acid; Beh: behenic acid; Lig: lignoceric acid; S: total saturated fatty acids; MUS: total monounsaturated fatty acids; PUS: total polyunsaturated fatty acids; US: total unsaturated fatty acids; PUS/S: total polyunsaturated to saturated fatty acids; O/L: oleic to linoleic acid ratio; SY: seed yield (kg/ha); OY: oil yield (Kg/ha).

\section{Result and Discussion}

Coheritability and genetic advance for oil traits and seed yield is shown in Table 1 . The range of coheritability was from $41 \%$ (between PUS/S and SY) to 98\% (between arachidic acid and eicosenoic acid). All oil traits have shown medium to high coheritability with oil content, oil quality concerning $\mathrm{O} / \mathrm{L}$ ratio, and SY. In this study, the positive coheritability estimate was observed between oil content, oil quality, and SY. This suggests that selection of either of the traits would simultaneously affect the SY, oil content or oil yield, and oil quality positively. SY has shown the highest coheritability and high genetic advance with both oil content and oil quality traits, even if higher for oil content than for oil quality. Oil content has recorded high coheritability and genetic advance with most of the evaluated traits including stearic acid, arachidic acid, eicosenoic acid, behenic acid, lignoceric acid, O/L ratio, OY, and SY. However, oil content has shown medium to low genetic advance with IV, palmitic acid, oleic acid, linoleic acid, S, MUS, US, PUS, and PUS/S. On the other hand, the O/L ratio (oil quality trait) has shown high coheritability and genetic advance with SY, OY, and most of the other oil quality traits. However, the $\mathrm{O} / \mathrm{L}$ ratio has scored a low genetic advance with low oil quality traits including palmitic acid, linoleic acid, and PUS. Both SY and OY have shown high coheritability and genetic advance with most yield-related agromorphological traits and high oil quality traits, but low genetic advance with low oil quality traits like linoleic acid, PUS, and PUS/S.

Table 2 shows the coheritability and genetic advance for oil content and oil quality and agromorphological traits. The range of coheritability was from $36 \%$ (between NSP with SWP and SHP to 96\%) (between NBP and AGBP). All evaluated agromorphological traits have shown medium to high coheritability with oil content, oil quality, and SY. A positive coheritability estimate was observed between oil content, oil quality, and SY traits and all other evaluated oil traits in the present study. SY has shown high coheritability and genetic advance with most of the agromorphological traits.

Oil content has shown medium to high coheritability and genetic advance with NMP, NBP, AGBP, PWP, 100SW, and NSPOD, but low genetic advance with NSP and SWP. $\mathrm{O} / \mathrm{L}$ ratio (major oil quality parameter) has shown high coheritability and genetic advance with NMP, NBP, AGBP, PWP, SWP, and 100SW but low genetic advance with NSPOD. These suggest that selection of either of the trait would simultaneously affect the desired trait positively. SY has shown the highest coheritability and genetic advance with OY and high oil quality traits like O/L ratio and MUS than with oil content and low oil quality traits, linoleic acid, PUS, PUS/S, and IV, showing that breeding for both oil yield and oil quality can be synchronized with seed yield (SY).

The coheritability and genetic advance (Table 2) for oil content, $\mathrm{O} / \mathrm{L}$ ratio, and SY and/or oil content, oleic acid, and SY is greater than those of oil content, linoleic acid (low oil quality trait), and SY or oil content, PUS/S, and SY can be further evidence showing that both oil content and oil quality can be improved with both seed yield and oil yield and there is the possibility of making selection for genotypes with high oil content, oil yield, and oil quality as well as high seed yield. OY has shown a similar trend with SY. Few studies are found for the coheritability of oil traits, e.g. de Reggi [29], Shrivastava and Jain [30], Yadav [31], and Mehta [32] found high estimates of coheritability for seed yield and oil traits in soybean. 
TABLE 2: Coheritability (upper row) with genetic advance (lower row) for agromorphological and oil traits.

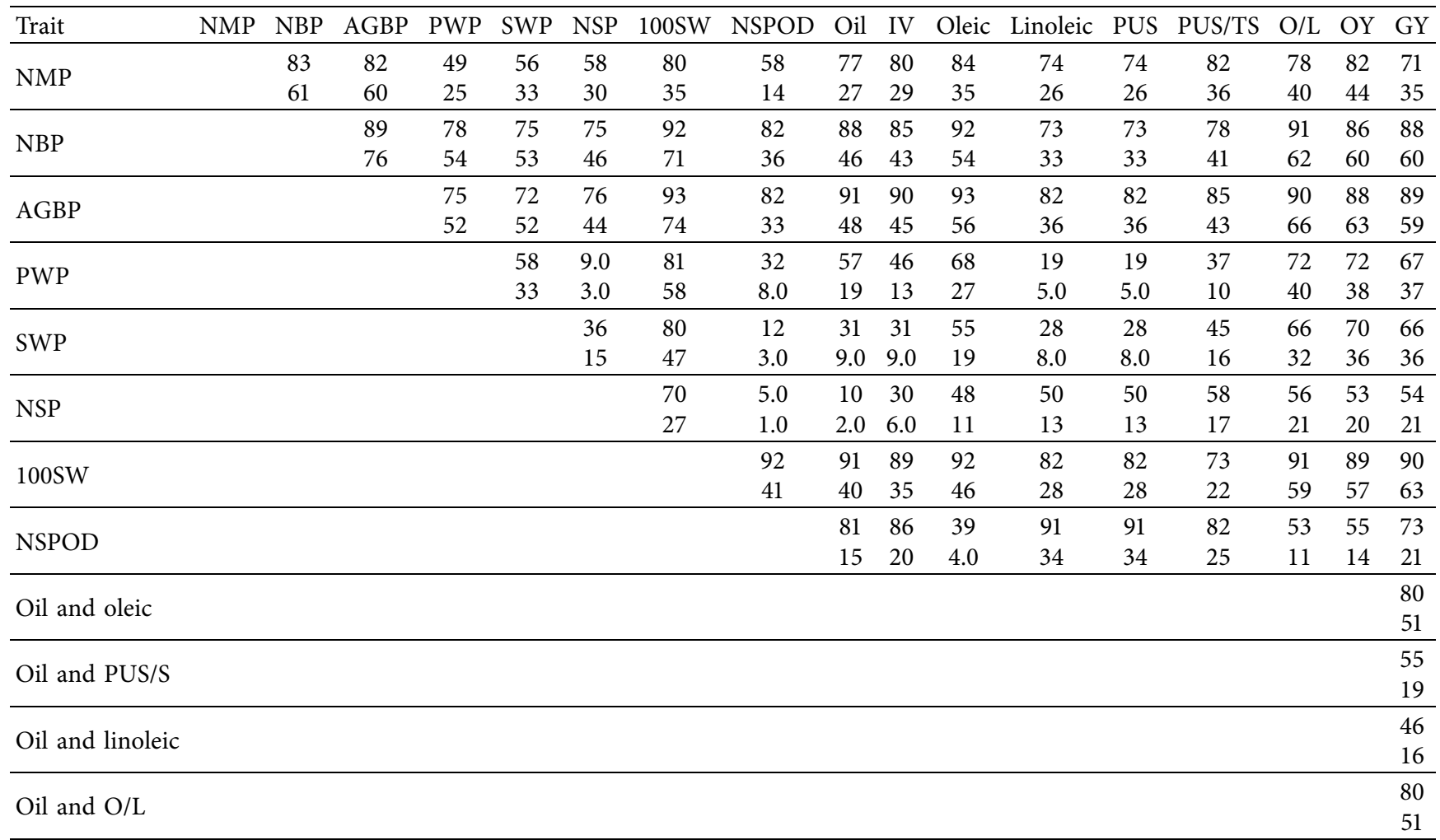

NMP: number of mature pod per plant; NBP: number of primary branches per plant; AGBP: above-ground biomass per plant; PWP: pod weight per plant; SWP: seed weight per plant; NSP: number of seeds per plant; SHP: shelling percent; 100SW: 100 seed weight; HI: harvest index; NSPOD: number of seeds per pod; O/L: oleic to linoleic acid ratio; OY: oil yield; SY: seed yield (kg/ha).

\section{Conclusion}

Since separate breeding for seed yield and oil traits is not cost-effective, it is better to synchronize two or more important traits in oil crops. The present study has shown the extent of coheritability of oil traits such as oil yield, oil content, and oil quality with seed yield and its related agromorphological traits. The estimates of coheritability and response to selection help breeders to conduct breeding for oil traits via making selection for agromorphological traits.

\section{Data Availability}

The raw data used to support the findings of this study are included within the supplementary material file.

\section{Ethical Approval}

The ethical approval is not applicable for this manuscript since it has no animal experiment according to Haramaya University's ethical committee.

\section{Conflicts of Interest}

The authors declare no conflicts of interest.

\section{Authors' Contributions}

Zekeria Yusuf did field experiment, collected the data, and analyzed the study; Habtamu Zeleke initiated and designed the study; Arno Hugo was responsible for acquisition of data and doing the laboratory phase; Wassu Mohammed and Shimelis Hussein analyzed and interpreted of data. All authors contributed to drafting the article and revising it critically for important intellectual content.

\section{Acknowledgments}

The authors are grateful to Haramaya University Research Office for their financial support and laboratory facility. This project was funded by Haramaya University Research Grant, under project code: HUIF_2019_06_01_110.

\section{Supplementary Materials}

The data used to support the findings of this study. (Supplementary Materials)

\section{References}

[1] A. L. Singh, "Phenology of groundnut," in Advances in Plant Physiology, A. Hemantranjan, Ed., vol. 6, pp. 295-382, Scientific Publishers (India), Jodhpur, India, 2003. 
[2] P. Janila, S. N. Nigam, M. K. Pandey, P. Nagesh, and R. K. Varshney, "Groundnut improvement: use of genetic and genomic tools," Frontiers in Plant Science, vol. 4, no. 23, p. 1, 2013.

[3] C. O. Ojiewo, P. Janila, P. Bhatnagar-Mathur, P Pandey, M K. Okori, and H Desami, "Advances in crop improvement and delivery research for nutritional quality and health benefits of groundnut (Arachis hypogaea L.)," Frontiers in Plant Science, vol. 11, no. 29, 2020.

[4] P. Janila, T. Radhakrishnan, T. V. Murali et al., High Oleic Peanuts for Asia and Africa to Meet the Needs of the Food Processing Industries, Case Study: Science forum, Stellenbosch, South Africa, 2018.

[5] D. B. Deshmukh, B. Marathi, H. K. Sudini et al., "Combining high oleic acid trait and resistance to late leaf spot and rust diseases in groundnut (Arachis hypogaea L.)," Frontiers in Genetics, vol. 11, p. 514, 2020.

[6] B. Nawade, G. P. Mishra, T. Radhakrishnan et al., "High oleic peanut breeding: achievements, perspectives, and prospects," Trends in Food Science \& Technology, vol. 78, pp. 107-119, 2018.

[7] C. Alemayehu, A. Berhanu, T. Mulugeta, M. Abdi, A. Tameru, and S. Helge, "Opportunities and constraints of groundnut production in selected drylands of Ethiopia," Drylands Coordination Group, vol. 74, 2014.

[8] E. S. Lander and N. J. Schork, "Genetic dissection of complex traits," Science, vol. 265, pp. 2037-2048, 1994.

[9] H. W. Johnson, H. F. Robinson, and R. E. Comstoks, "Estimates of genetics and environmental variability in soybean," Agronomic Journal, vol. 45, pp. 374-382, 1955.

[10] S. Iqbal, T. Mahmood, M. Ali, M. Anwar, and M. Sarwar, "Path coefficient analysis in different genotypes of soyabean (Glycine max L.)," Pakistan Journal of Biological Sciences, vol. 6, pp. 1085-1087, 2003.

[11] M. M. Rohman, A. S. M. Iqbal, M. S. Arifin, Z. Akhtar, and M. Husanuzzaman, "Genetic variability, correlation and Path analysis in Mungbean," Asian Journal of Plant Scinces, vol. 2, no. 17-24, pp. 1209-I211, 2003.

[12] M. Nei, Studies on the Application of Biometrical Genetics to Plant Breeding, Memoirs of the College of Agriculture Kyoto University, Kyoto, Japan, 1960.

[13] D. S. Falconer and T. F. C. Mackay, Introduction to Quantitative Genetics, Longmans Green, Harlow, Essex, UK, 4th edition, 1996.

[14] J. Vasquez-Kool, "Coheritability and coenvironmentability as concepts for partitioning the phenotypic correlation," 2019.

[15] M. S. Akhtar, Y. Oki, T. Adachlt, and M. H. R. Khan, "Analyses of the genetic parameters (variability, heritability, genetic advance, relationship of yield and yield contributing characters) for some plant traits among Brassica cultivars under phosphorus starved environmental cues," International Journal of Environmental Science and Technology, vol. 112, no. 1, pp. 91-98, 2007.

[16] D. K. Makan and S. S. Saini, "Coheritability variation in rice," Indian Journal of Genetics and Plant Breeding, vol. 42, no. 3, pp. 296-301, 1982.

[17] S. Kumar and D. K. Dubbey, "Variability, heritability and correlation studies in grasspea (Lathyrus sativus L.)," Lathyrus Lathyrism Newsletter, vol. 2, pp. 79-81, 2001.

[18] P. E. Melton, S. Rutherford, V. S. Voruganti et al., "Bivariate genetic association of KIAA1797 with heart rate in American Indians: the strong heart family study," Human Molecular Genetics, vol. 19, no. 18, pp. 3662-3671, 2010.
[19] Y. Jia and J. L. Jannick, "Multiple-trait genomic selection methods increase 1248 genetic value prediction accuracy," Genetics, vol. 192, pp. 1513-1522, 2012.

[20] R. Cheng, J. Borevitz, and R. W. Doerge, "Selecting informative traits for multivariate quantitative trait locus mapping helps to gain optimal power," Genetics, vol. 195, pp. 683-691, 2013.

[21] J. Folch, M. Lees, and G. H. Sloane-Stanley, "A simple method for the isolation and purification of total lipids from animal tissue," Journal of Biological Chemistry, vol. 226, pp. 497-509, 1957.

[22] AOAC, Official Methods of Analysis, Association of Official Analytical Chemists, Arlington, VA, USA, 15th edition, 1990.

[23] R. R. Comstock and H. F. Robinson, "Genetic parameters, their estimation and significance,"vol. 1, pp. 248-291, in Proceedings of the 6th international Grassland Congress, vol. 1, pp. 248-291, National publishing Co., Washington, DC, USA, August 1952.

[24] R. W. Allard, Principles of Plant Breeding, John Wiley and Sons, New York NY, USA, 1960.

[25] R. K. Singh and B. D. Chaudhury, Biometrical Methods in Quantitative Genetic Analysis, Kalyani publication, New Delhi, India, 1979.

[26] H. W. Johnson, H. F. Robinson, and R. E. Comstoks, "Estimates of genetics and environmental variability in soybean," Agronomy Journal, vol. 45, pp. 374-82, 1955.

[27] SAS Institute, SAS Enterprise Guide, Version 9.2, SAS Institute, Cary, NC, USA, 2011.

[28] R. G. O’Brien, “A simple test for variance effects in experimental designs," Psychological Bulletin, vol. 89, pp. 570-574, 1981.

[29] C. de Reggi, "Generalization of the notion of heritability of several characters: coheritability," Annals of Human Genetics, vol. 15, pp. 41-44, 1972.

[30] A. N. Shrivastava and J. K. Jain, "Variability and coheritability estimates for physiological and economic attributes in soybean," Indian Journal of Genetics, vol. 54, no. 2, pp. 179-183, 1994.

[31] V. Yadav, "Genetic variability and coheritability estimates in soybean (Glycine max. L.)," International Journal of Plant Sciences, vol. 2, no. 1, pp. 9-11, 2007.

[32] A. R. Mehta, "Assessment of genetic variability, coheritability and divergence in Soybean," Jawaharalal Nahru Krishi Vishwa Vidyalaya, Jabalpur, India, M.Sc Thesis. 